\title{
Notas sobre o Grupo de Galilei: Aspectos Geométricos e Recentes Desenvolvimentos
}

\author{
Notes on the Galilei Group: Geometric Aspects and Recent Developments
}

\author{
Luciano M. Abreu* \\ Centro de Ciências Exatas - UFRB \\ Cruz das Almas - Bahia - 40111-340 \\ Ademir E. Santana情 \\ Instituto de Fúsica - UnB \\ Brasília - DF - 70910-900 \\ Esdras S. Santos e Aurino Ribeiro Filho \\ Instituto de Física - UFBA \\ Campus Federação - Ondina, \\ Salvador - Bahia - 40210-340
}

Apresentamos uma revisão pedagógica sobre as simetrias galileanas, seus desenvolvimentos e o interesse atual na geometria do espaço-tempo não-relativístico para fins de aplicação. Inicialmente tratamos de alguns aspectos históricos e seguimos com a definição do grupo de Galilei; nos concentramos depois na noção de covariância Galileana. Enfatizamos o estudo de representações que levam a equações da mecânica quântica não-relativística, como a equação de Schrödinger para partículas de spin zero e a equação de Pauli-Schrödinger para partículas de spin 1/2. A forma como as representações são construídas torna evidente, para fins pedagógicos, que conceitos como o spin, não são efeitos tipicamente relativísticos, como aparecem em alguns textos sobre mecânica quântica. Por outro lado, reforçamos a importância da estrutura tensorial (geométrica) para aplicações em sistemas não-relativísticos, como no caso da física da matéria condensada.

Palavras-chaves: Grupo de Galilei, Equação de Schrödinger, Estrutura Tensorial.

We present a pedagogical review on the Galilean symmetries, their developments and the current interest about the geometry of non-relativistic space-time for application of. Initially we treat some historical aspects and follow with the definition of Galilei group; focus after in the notion of Galilean covariance. We emphasize the study of representations that lead to equations of non-relativistic quantum mechanics, such as Schrödinger equation for particles of spin zero and Pauli-Schrödinger equation for particles of spin $1 / 2$. The way the representations are builded makes clear, for pedagogical purposes, that concepts such as spin are not typically relativistic effects, as they appear in some textbooks about quantum mechanics. On the other side, we reinforce the importance of tensor (geometric) structure for applications in non-relativistic systems, as in the case of condensed matter physics.

Key-words: Galilei Group, Schrödinger Equation, Tensor Structure.

\footnotetext{
${ }^{\dagger}$ Endereço Atual: Department of Physics, University of

Alberta, T6G2J1, Edmonton, AB, Canada.

*Endereço Eletrônico: abreulm@uol.com.br

‡Endereço Eletrônico: asantana@fis.unb.br
} 


\section{INTRODUÇÃ̃O}

O conceito de simetria vem sendo edificado pela humanidade desde tempos imemoriais e tem sido abordado de um ponto de vista filosófico, artístico e matemático. O conceito se aplica a uma vastidão de situações de interesses estéticos e utilizáveis, e é disso que resulta a procura por sua caracterização e estudo. Para Weyl [1], em uma elegante síntese do pensamento ocidental, simetria significa:

...[symmetry is] something like well-proportioned, well-balanced, and symmetry denotes that sort of concordance of several parts by which they integrate into a whole.

Além disso, a natureza da simetria é descrita como:

... One may ask whether the aesthetical value of symmetry depends on its vital value. ... I am inclined to think with Plato that the mathematical idea is the commom origin of both [ways]: the mathematical laws governing nature are the origin of symmetry in nature, the intuitive realization of the idea in the creative artist's mind its origin in art.

A geometrização do conceito de simetria garantiu ferramentas próprias aos antigos arquitetos, e o ápice deste acúmulo de conhecimentos é o surgimento, no século XVII, da engenharia de construção, que se funda a partir das equações de movimento da mecânica Newtoniana. Ainda a partir da geometria Euclidiana o conceito de grupo é introduzido, e é particularmente desenvolvido por Galois, fornecendo uma caracterização matemática ao conceito de simetria das formas regulares no espaço, como o quadrado, cubo, etc. E assim aparece o grupo de pontos [2].

Um aspecto fascinante neste trajeto histórico acontece em outro momento crucial: a descoberta por Sophus Lie da estrutura de grupos contínuos associada às equações diferenciais, das quais as equações de movimento são um caso particular, por envolver as coordenadas espaço-temporal [3 7]. Um dos principais resultados do trabalho de Lie foi mostrar que o estudo das simetrias subjacentes a uma dada equação diferencial permite que sejam encontradas as classes de suas soluções. Desse modo a Física do Século XX apoderou-se definitivamente sobre o conceito de simetria, descrito matematicamente a partir da estrutura de grupo [8].

O estudo das simetrias das equações de movimento deu origem, então, aos denominados grupos cinemáticos, ou seja, os grupos de simetrias do espaço-tempo. No caso da chamada física não-relativística, tanto clássica como quântica, essas simetrias formam o grupo de Galilei. De modo outro, temos o grupo de Lorentz, que estrutura as transformações cinemáticas da física relativística, deixando invariante por exemplo as equações de Maxwell [8, 9].

Ao explorar o sentido inverso dos resultados de Lie, ou seja, ao estudar as representações irredutíveis do grupo de Lorentz, Wigner em 1939 deduz, por exemplo, a equação de KleinGordon e a equação de Dirac [10]. Essas representações possibilitou então uma classificação das partículas elementares. Cada representação da simetria cinemática fornece uma equação de movimento (uma equação diferencial) que pode ser associada às partículas elementares [11, 13]. No estudo de Wigner, surgem apenas as representações associadas à natureza do spin e da massa das partículas. Com o desenvolvimento da física das partículas elementares, outros rótulos quânticos são introduzidos, como o isospin e as cores. De um ponto de vista teórico isso levou às chamadas teorias de campos de calibre não-abeliano, baseadas na invariância da teoria por grupos de calibre (gauge, em inglês). O exemplo mais conhecido desse tipo de formalismo é a teoria eletromagnética, descrita por um grupo de calibre denominado $U(1)$. As teorias de calibre remontam o início do século XX com os trabalhos pioneiros de Weyl, ao tentar descobrir um conteúdo geométrico para interação eletro- 
magnética, como havia feito Einstein com a gravitação. Em meados da Década de 1950 Yang e Mills 14 publicam um trabalho, onde utilizam o $S U(2)$ como grupo de calibre, para tentar descrever os mésons e píons, que àquela época eram consideradas, juntamente com o próton e o neutron, como partículas elementares. Os grupos de calibres descrevem as interações elementares da natureza, que usualmente são referidas como quatro: a força gravitacional, que descreve a estabilidade das formações no cosmos; a força eletromagnética, descrevendo a estrutura eletrônica dos átomos, moléculas e seus agregados, ou seja, regulando os processos químicos e biológicos; a força fraca, responsável pelos processos de decaimento nuclear; e a força forte, responsável pela estabilidade do núcleo. Na década de 1960, Weinberg, Salam e Glashow construiram uma teoria de calibre onde unificaram em um mesmo objeto físico as forças eletromagnética e a fraca, dando origem à interação eletrofraca. Um feito similar havia sido alcançado por Maxwell um século antes, quando unificou a força elétrica com a magnética, resultando no campo eletromagnético.

A despeito do avanço que se sucedeu ao trabalho de Wigner no entendimento do grupo de Poincaré, é somente na década de 1950 que se inicia um estudo similar para a mecânica quântica não-relativista. Nesse caso, a estrutura de simetria fica estabelecida pela invariância da equação de Schrödinger por transformações no espaço e no tempo, que resulta no grupo de Galilei.

As representações unitárias de um grupo de simetria são deduzidas através da associação de cada elemento do grupo a um operador unitário satisfazendo as regras de composição do grupo [15, 16]. Mais especificamente, considere $\mathrm{G}$ um grupo de simetria com elementos $a, b, c, \ldots$ Para cada $a \in \mathrm{G}$ está associado um operador unitário $U(a)$ definido num espaço de Hilbert, tal que $U(a) U(b)=e^{\phi(a, b)} U(a, b)$. O fator de fase $\phi(a, b)$ é uma função de $a$ e $b$ real e contínua. Se $\phi=0$, a representação é dita fiel. Caso contrário, a representação é dita projetiva. Neste caso, um vetor no espaço de Hilbert fica definido a menos do fator de fase.
Inönü e Wigner [17, estudando as representações unitárias do grupo de Galilei, concluiram que com as representações fieis não seria possível a construção de funções de onda - estados - que fossem localizadas, e que tivessem velocidades definidas. $\mathrm{Ou}$ seja, tais representações devem ser descartadas enquanto possíveis candidatas para descrever uma partícula quântica não-relativista. Bargman [18] mostrou, por outro lado, que para o grupo de Rotação, Lorentz e Poincarè, as representações projetivas poderiam ser reduzidas a uma representação fiel. Entretanto, isto não se dá com o grupo de Galilei, onde representações projetivas, em geral, não são redutíveis a uma representação fiel [19]. Em uma síntese dos trabalhos de Iönü, Wigner e Bargman, Hamermesh [20], estudando a álgebra de Lie do grupo de Galilei, estabeleceu que operadores de posição e momento poderiam ser definidos somente para o caso de representações projetivas, que passaram a ser também denominadas de representações físicas [21]. Desde então, o interesse nas representações unitárias descrevendo simetrias galileanas tem-se concentrado, principalmente, e como acontece com o grupo de Poincaré, no estudo de estruturas de simetrias conformes e internas 22 31. Deve ser enfatizado que na análise de simetrias internas, as variáveis de spin aparecem por consistência na física nãorelativista, e não como uma propriedade exclusiva da invariância Lorentziana. Isso se dá mesmo para sistemas clássicos, há muito conhecido, mas pouco divulgado e entendido [31. Sistemas não-lineares têm sido analisados 32, 33] e, no mesmo contexto de simetrias, vários desenvolvimentos e aplicações na teoria quântica de campos a temperatura finita têm sido implementados [34 40].

A importância das simetrias galileanas para a matéria condensada é reconhecida a longo tempo. Um exemplo importante aparece na análise das excitações elementares em superfluidos. Neste caso, tais excitações, introduzidas e chamadas de fônons e rótons por Landau [41, são interpretadas como manifestações coletivas do campo de Schrödinger. Ou seja, o campo original (e ressalte-se, invariante pelas 
transformações do grupo de Galilei) se rearranja, devido às interações, gerando tais excitações. O resultado final é uma quebra de simetria Galileana. De fato, no caso da componente do campo de fônons caracterizada por uma velocidade de fase $c_{s}$, a Lagrangeana é invariante sob uma transformação de Lorentz, onde a velocidade da luz é substituida por $c_{s}$. Assim, surgem as famosas relações de dispersão para os fônons e rótons, similares às relações energia-momento no caso relativista. Esta invariância Lorentziana das excitações, porém, só possui um significado matemático, no sentido de que a interpretação física do espaco-tempo onde a teoria está formulada não se reduz àquela correspondente ao espaço de Minkowski, para o qual a velocidade da luz tem um carácter universal. Esta quebra de simetria, do campo original Galileano para o resultado final Lorentziano, é ainda acompanhada de outros fatos em conexão com as transformações de Galilei: bem conhecido é, na teoria de Landau, a importância do movimento de translação do fluido, descrito por uma transformação pura de Galilei, e suas interações com impurezas e paredes do recipiente. Ou seja, a superfluidez é um fenômeno característico do regime de baixas velocidades, isto é, da cinemática Galileana, e para sua compreensão plena e futuros desenvolvimentos demanda o aprimoramento da teoria de campos Galileana.

É neste sentido que Takahashi [42 44] introduziu uma versão covariante para o grupo de Galilei baseada em tensores penta-dimensionais [45 48]. Esse método foi utilizado para a dedução de equações de campo não lineares, a partir das quais o rearranjo de simetrias em superfluidos tem sido analisado em conexão com bósons de Goldstone. Desde então, a noção de covariância Galileana tem sido desenvolvida em diversas linhas, tratando de problemas gravitacionais e cosmológicos [49 54 à teoria de partículas de spins altos em física da matéria condensada [55]. Um dos objetivos aqui é apresentar a estrutura covariante do grupo de Galilei, enfatizando a natureza do espaço vetorial métrico onde as transformações estão definidas [16]. Neste sentido, primeiro é estabelecido que o grupo de Galilei pode ser tratado como um grupo de transformações lineares sobre uma variedade, a ser denominada por $\mathcal{G}$, na qual a métrica é conservada. Sob este ponto de vista de grupo de transformação, fica a apresentação do grupo de Galilei unificada com as de outros grupos importantes na Física, como o grupo de Rotações, onde a variedade é o espaço Euclidiano $(\mathbb{E})$; ou ainda, os grupos de Lorentz e Poincaré, onde a variedade é o espaço de Minkowski. Segundo, é alcançado transparência na apresentação, pois o conteúdo físico das cinco dimensões do espaço $\mathbb{G}$ fica definido desde início. De fato, 3 das 5 dimensões estarão relacionadas ao espaço $\mathbb{E}$, enquanto que as outras estarão vinculadas à velocidade e ao tempo. Além disso, $\mathbb{G}$ pode ser definido por um processo de imersão de $\mathbb{E}$ em um espaço espaço de de Sitter do tipo $(4+1)$. Esta relação entre tais espaços métricos possui importância na definição apropriada da álgebra de Clifford, quando do estudo de representações de spin semi-inteiros. Por outro lado, existe uma associação dessa geometria de $\mathbb{G}$ com espaços anti-de Sitter $(3+2)$ e o grupo de transformações conformes. Isto permite o desenvolvimento de teorias do tipo AdS/CFT (anti-de Sitter e CFT do inglês: Conformal Field Theory) [56-58] na análise de sistemas de spins fortemente acoplados, como no caso (experimental) de átomos fermiônicos resfriados [59, 60].

A apresentação está organizada da seguinte forma. Na Sec. 2, apresentamos alguns elementos históricos sobre as simetrias de Galilei. Seguimos, na Secs. 3 e 4, com as definições do grupo de Galilei, e nas Secs. 5 e 6 tratamos da equação de Schrödinger para a partícula livre de spin zero e $1 / 2$, respectivamente. Nas Secs. 7 e 8 consideramos a noção de covariância Galileana e suas representações, seguindo a Ref. [16] e uma apresentação pedagógica. Na Sec. 9 estão as conclusões e observações finais.

\section{PRINCÍPIO DA RELATIVIDADE GALILEANA: NOTAS HISTÓRICAS}

Na edição inglesa da obra intitulada " $D i$ - 
alogue Concerning the Two Chief World Systems" 61], pp. 186-187, Galilei através de seu alter-ego Salviati comenta:

Shut yourself up with some friend in the main cabin below decks on some large ship, and we have with you there some flies, butterflies, and other small flying animals. Have a large bowl of water with some fish in it; hang up a bottle that empties drop by drop into a wide vessel beneath it. With the ship standing still, observe carefully how the little animals fly with equal speed to all sides of the cabin. The fish swim indifferently in all directions; the drops fall into the vessel beneath; and, in throwing something to your friend, you need throw it no move strongly in one direction than another, the distances being equal, jumping with your feet together, you pass equal spaces in every direction.

When you have observed all these things carefully (though there is no doubt that when the ship is standing still everything must happen in this way), have the ship proceed with any speed you like, so long as the motion in uniform and not fluctuating this way and that. You will discover not the least change in all the effects named, nor could you tell from any of them whether the ship was moving or standing still...

Com estas palavras nascia a denominada relatividade galileana, cujo impacto na modernidade foi imenso e ajudou Galilei a ser considerado, por esta e outras contribuições científicas 61]-65], como o fundador da física como ciência e também o primeiro físico moderno. De fato, Sobel [66, 67], Claret [68, e Krudsen and Hjorth [69] indicam tal trecho no mencionado diálogo como precursora da teoria física [70].
A mencionada obra de Galilei veio a lume em fevereiro de 1632 [71, publicada em Florença, apesar de tê-la iniciado quatro anos antes, e nela o seu autor defende a teoria copernicana (heliocentrismo). Tal feito incitou os seus inimigos a denunciar a obra como transgressora do decreto de fevereiro de 1616, em que a Sagrada Congregação do Index havia proibido a teoria do cônego Copérnico [72, 73]. Este fato implicou na sua proibição em agosto, do mesmo ano, além da intimação à Galilei, em setembro, a fim de comparecer diante do comissário geral do Santo Ofício, em Roma. Ressalte-se que o verdadeiro título desta obra é "Diálogo de Galileu Galilei linceu, matemático extraordinário do Estúdio de Pisa e filósofo e matemático primário do Sereníssimo GrãoDuque da Toscana; onde, nas reuniões de quatro jornadas, discorre-se sobre os dois máximos sistemas do mundo - ptolemaico e copernicano - propondo de maneira indeterminada as razões filosóficas e naturais tanto para uma quanto para a outra parte" [73, 76]. Neste livro estão transcritos quatro diálogos, em que o seu autor imaginou que em cada dia diferente era desenvolvido um dos diálogos entre três personagens Fillippe Salviati, Simplício e Giovan Francesco Sagredo. O primeiro, seguidor e defensor ferrenho do copernicanismo é, no fundo, o próprio Galileu; o segundo interlocutor é uma figura imaginária que se apresenta como um defensor das teorias de Ptolomeu e, finalmente, Sagredo que é apresentado como alguém dotado de abertura intelectual suficiente para escutar e ponderar sobre as duas concepções do universo defendidas pelos outros dois debatedores.

Dos quatro diálogos, aquele que nos interessa mais de perto, nesta nota, é o do segundo dia (ou segunda jornada), onde os três interlocutores dedicam-se à discussão sobre o movimento da Terra. Na tentativa de calar as críticas dos seguidores da doutrina ptolemaica, o autor do citado livro introduz "neste dia", dois dos princípios fundamentais da física e que se tornaram básicos na mecânica newtoniana: o princípio da inércia e o princípio da relatividade. De acordo com diferentes autores, a noção de "inércia" já havia despertado 
o interesse de antecessores de Galileu, entretanto, foi ele o primeiro a compreender, de fato, o conteúdo de tal princípio, apesar de não tê-lo enunciado, e sim aplicado o mesmo com bastante rigor científico, utilizando-se para isso de base experimental.

O princípio da relatividade (ou princípio da relatividade clássica de Bruno-Galileu-Newton [77], ou princípio da relatividade galileana) foi lançado por Galilei a fim de responder às críticas sobre a possibilidade de existência da rotação terrestre. Para os seguidores do aristotelismo, àquela época, os fenômenos mecânicos ocorriam como se a Terra fosse fixa. Para dirimir tais dúvidas, Galilei se utilizaria do seu personagem Salviati, no trecho do livro já citado antes, a fim de explicitar o fato de que não é possível, no interior de um sistema, inferir-se por meios de experimentos mecânicos, se o sistema em estudo está em repouso ou em movimento retilíneo uniforme. Com tal afirmação é introduzida a relatividade de Galileu, centrada nas transformações galileanas que, duzentos e setenta e três anos mais tarde viria ser entendida como um caso particular da relatividade especial de Einstein.

A exemplo do que acontece com a verdadeira origem do princípio da inércia, alguns autores [73, 78] conjecturam que o princípio da relatividade clássica tenha sido introduzido, indiretamente, por Giordano Bruno [79] e por Nicole d'Oresme 80, que, no século XIV haviam descoberto a importância do movimento relativo entre objetos, quando escreveu que os movimentos diurnos dos céus podem ser igualmente explicados pela rotação diurna da Terra.

O princípio da relatividade, junto as rotações espaciais e com as translações no espaço e no tempo formam um grupo de simetria, conhecido como grupo de Galilei, a partir do qual toda a física galileana pode ser construída a partir da análise das diferentes representações. Este resultado leva a consequências, como já frisado, pouco compreendidas e exploradas na física não-relativística, como o aparecimento da noção de spin associado à equação de Schrödinger ou a física clássica, sem apelo à teorias relativísticas. Nas próximas seções apresentamos um resumo dos principais resultados ao longo desta linha.

\section{O GRUPO DE GALILEI}

As transformações de Galilei nãohomogêneas, tidas como as transfomações das coordenadas $(\mathbf{x}, t)$ de um evento no espaço e no tempo para outras coordenadas $(\overline{\mathbf{x}}, \bar{t})$, são definidas da seguinte forma

$$
\begin{aligned}
\overline{\mathbf{x}} & =R \mathbf{x}+\mathbf{v} t+\mathbf{a}, \\
\bar{t} & =t+b,
\end{aligned}
$$

com $\mathbf{x}$ sendo as coordenadas espaciais no espaço Euclidiano tridimensional $\mathbb{E}=\mathbb{R}^{3}$ e $t$ a coordenada temporal; $R$ descreve uma rotação em $\mathbb{E} ; \mathbf{v}$ prescreve a mudança do sistema de referência, isto é, a transformação pura de Galilei; a uma translação espacial e $b$ uma translação temporal. As equações (1) e (2) estabelecem a relação entre dois referenciais inerciais da física não-relativística. Denotamos essas transformações por

$$
G(\mathbf{x}, t)=(\overline{\mathbf{x}}, \bar{t})
$$

com $G$ dado por

$$
G=(b, \mathbf{a}, \mathbf{v}, R),
$$

sendo um objeto que atua nas coordenadas de acordo com as equações (1) e (2). Considerando duas transformações $G_{1}=$ $\left(b_{1}, \mathbf{a}_{1}, \mathbf{v}_{1}, R_{1}\right)$ e $G_{2}=\left(b_{2}, \mathbf{a}_{2}, \mathbf{v}_{2}, R_{2}\right), \quad$ a aplicação sucessiva de duas destas, $G_{2} G_{1}$, resulta em uma lei de composição dada por

$$
\begin{aligned}
G_{2} G_{1}= & \left(b_{2}+b_{1}, \mathbf{a}_{2}+R_{2} \mathbf{a}_{1}+b_{1} \mathbf{v}_{2}, \mathbf{v}_{2}\right. \\
& \left.+R_{2} \mathbf{v}_{1}, R_{2} R_{1}\right) .
\end{aligned}
$$

Ou seja, temos outra transformação de Galilei, $G=G_{2} G_{1}$. A transformação identidade é

$$
E=(0,0,0,1)
$$

e a inversa de $G$ é

$$
G^{-1}=\left(-b,-R^{-1}(\mathbf{a}-b \mathbf{v}),-R^{-1} \mathbf{v}, R^{-1}\right) .
$$


Como a associatividade também vale para a composição definida na Eq. (5), o conjunto de elementos $G$ dados na Eq. (4) formam um grupo chamado grupo de Galilei $(\mathcal{G})$, que é o grupo de simetria da física nãorelativística. Este é um grupo especificado por dez parâmetros: três descrevendo as rotações (os três ângulos de Euler, por exemplo) e definindo a matriz $R$; três definindo a transformação pura de Galilei, ou seja v; três descrevendo as translações espaciais, ou seja a; e um parâmetro para descrever as translações no tempo, ou seja $b$. Temos então um grupo a 10 parâmetros. As equações (1) e (2) são sua autorepresentação, ou representação de definição.

Podemos reconhecer de imediato alguns dos subgrupos de $\mathbf{G}$ :

$$
\begin{aligned}
\mathcal{T}= & \{(b, 0,0,1)\}, \text { o subgrupo das translações } \\
& \text { temporais; }
\end{aligned}
$$

$\mathcal{S}=\{(0, \mathbf{a}, 0,1)\}$, o subgrupo das translações espaciais;

$$
\begin{aligned}
\mathcal{B}= & \{(0,0, \mathbf{v}, 1)\}, \text { o subgrupo das trans- } \\
& \text { fomações puras; }
\end{aligned}
$$

$\mathcal{R}=\{(0,0,0, R)\}$, o subgrupo das rotações.

O subgrupo $\mathcal{T} \times \mathcal{S}$ forma um subgrupo abeliano invariante, e $\mathcal{G} /(\mathcal{T} \times \mathcal{S})$ é isomórfico a $\mathcal{B} \times \mathcal{R}$, o chamado grupo de Galilei homogêneo $(\Gamma)$. Vamos obter a álgebra de Lie de $\mathcal{G}$, a qual denotaremos $\mathfrak{g}$, utilizando uma representação unitária irredutível sobre o espaço das funções dependentes de $(\mathbf{x}, t)$, i.e.

$$
U(b, \mathbf{a}, \mathbf{v}, R) f(\mathbf{x}, t)=f(\overline{\mathbf{x}}, \bar{t}) .
$$

Tomando $(b, \mathbf{a}, \mathbf{v}, R)$ com parâmetros infinitesimais, conseguimos os geradores do grupo $\mathcal{G}$ nesta representação, e podemos escrever

$$
U(b, \mathbf{a}, \mathbf{v}, R)=e^{b H} e^{\mathbf{a} \cdot \mathbf{P}} e^{\mathbf{v} \cdot \mathbf{k}} e^{\theta \cdot \mathbf{J}},
$$

onde a base de $\mathfrak{g}$ é

$$
\begin{aligned}
& H=i \frac{\partial}{\partial t} \text { é o gerador do subgrupo } T ; \\
& \mathbf{P}=-i \nabla \text { é o gerador de } S
\end{aligned}
$$

$$
\begin{aligned}
& \mathbf{J}=-i \mathbf{r} \times \nabla \text { é o gerador de } R ; \\
& \mathbf{k}=i t \nabla \text { é o gerador de } V .
\end{aligned}
$$

Deste modo, as relações de comutação que definem $\mathfrak{g}$ são

$$
\begin{aligned}
{\left[J_{i}, J_{j}\right] } & =i \varepsilon_{i j k} J_{k}, \\
{\left[J_{i}, K_{j}\right] } & =i \varepsilon_{i j k} K_{k}, \\
{\left[J_{i}, P_{j}\right] } & =i \varepsilon_{i j k} P_{k}, \\
{\left[J_{i}, H\right] } & =0, \\
{\left[K_{i}, K_{j}\right] } & =0, \\
{\left[K_{i}, P_{j}\right] } & =0, \\
{\left[K_{i}, H\right] } & =-i P_{i}, \\
{\left[P_{i}, P_{j}\right] } & =0, \\
{\left[P_{i}, H\right] } & =0,
\end{aligned}
$$

onde $\varepsilon_{i j k}$ é o tensor totalmente antissimétrico.

Os invariantes desta álgebra são

$$
\begin{aligned}
I_{1} & =\mathbf{P}^{2}, \\
I_{2} & =(\mathbf{K} \times \mathbf{P})^{2} .
\end{aligned}
$$

É possível mostrar que por meio do estudo de representaçoes unitárias deste grupo (ditas fiéis) não obtemos equações dinâmicas invariantes por $\mathcal{G}$ [17] consistentes para descrever partículas não-relativísticas. Isto seria esperado numa primeira abordagem, pois no caso do grupo de Poincaré, são as representações unitárias irredutíveis que descrevem as partículas relativísticas. As dificuldades com o grupo de Galilei começam neste ponto. Para conseguir representações unitárias irredutíveis com significado físico, temos que construir uma extensão central de $\mathcal{G}$, o que faremos a seguir.

\section{O GRUPO DE GALILEI ESTENDIDO}

Seja $\mathcal{G}$ o grupo de Galilei e $\xi$ uma função real em $\mathcal{G} \times \mathcal{G}$, que satisfaz

$$
\begin{aligned}
& \xi\left(G_{1}, G_{2}\right)+\xi\left(G_{1} G_{2}, G_{3}\right) \\
& \quad=\xi\left(G_{1}, G_{2} G_{3}\right)+\xi\left(G_{2}, G_{3}\right), \\
& \xi(E, E)=0,
\end{aligned}
$$


sendo $E$ dado pela Eq. (6) . Podemos definir um novo grupo, $\tilde{G}_{\xi}=\mathbb{R} \times \mathcal{G}$, cujos elementos denotaremos por $\tilde{G}=(\lambda, G), \lambda \in \mathbb{R}$ e $G \in \mathcal{G}$, com a lei de composição

$$
\left(\lambda_{1}, G_{1}\right)\left(\lambda_{2}, G_{2}\right)=\left(\lambda_{1}+\lambda_{2}+\xi\left(G_{1}, G_{2}\right), G_{1} G_{2}\right) .
$$

$\tilde{G}_{\xi}$ é dito ser uma extensão central de $G$ com respeito a $\xi$. Um subgrupo abeliano de $\tilde{G}_{\xi}$ é identificado de imediato, $\Lambda=$ $\{(\lambda, E) \mid \lambda \in \mathbb{R}\}$, que chamaremos de grupo de fase; e é isomórfico a $\mathbb{R}$.

Em geral, por convenção de notação, uma extensão central de uma álgebra de Lie com base $\left\{a_{1}, \ldots, a_{n}\right\}$ satisfaz a

$$
\begin{aligned}
& {\left[a_{i}, a_{j}\right]=c_{i j}^{k} a_{k}+\beta_{i j} a_{0},} \\
& {\left[a_{i}, a_{0}\right]=0,}
\end{aligned}
$$

onde $\left\{c_{i j}^{k}\right\}$ são as constantes de estrutura da álgebra, $a_{0}$ o vetor base da subálgebra do grupo de fase e $\beta_{i j}$ é dito ser o elemento central da álgebra estendida. Seguindo esta forma, vamos escolher a seguinte extensão central da Eq. (19) da álgebra $\mathfrak{g}$ :

$$
\left[K_{i}, P_{j}\right]=i m \delta_{i j} I
$$

sendo $I$ o operador unidade, que é o gerador do subgrupo $\Lambda$ e $m$ um número real. Posteriormente veremos que $m$ é associado com a massa. Os invariantes desta álgebra estendida são

$$
\begin{aligned}
M & =m I, \\
U & =H-\frac{\mathbf{P}^{2}}{2 m}, \\
S^{2} & =(\mathbf{J}-\mathbf{K} \times \mathbf{P})^{2} .
\end{aligned}
$$

Podemos agora explicitar a lei de composição dada pela Eq. 27) no caso particular da extensão central dada na Eq. (30). Consideremos então um elemento de $\tilde{G}_{\xi}$ na forma

$$
\tilde{G}=e^{i \lambda I} e^{b H} e^{\mathbf{a} \cdot \mathbf{P}} e^{\mathbf{v} \cdot \mathbf{k}} R
$$

onde $R$ representa as rotações. Logo a lei de composição é

$$
\tilde{G}^{\prime} \tilde{G}=e^{i \lambda^{\prime} I} e^{b^{\prime} H} e^{\mathbf{a}^{\prime} \cdot \mathbf{P}} e^{\mathbf{v}^{\prime} \cdot \mathbf{k}} R^{\prime} e^{i \lambda I} e^{b H} e^{\mathbf{a} \cdot \mathbf{P}} e^{\mathbf{v} \cdot \mathbf{k}} R .
$$

Da fórmula de Baker-Hausdorff,

$$
e^{-B} A e^{B}=A+[A, B]+\frac{1}{2}[[A, B], B]+\ldots,
$$

obtemos

$$
e^{-b H} \mathbf{v} \cdot \mathbf{k} e^{b H}=\mathbf{v} \cdot \mathbf{k}+b \mathbf{v} \cdot \mathbf{P} .
$$

Temos também as relações

$$
\begin{aligned}
e^{\mathbf{u} \cdot \mathbf{k}} e^{\mathbf{w} \cdot \mathbf{P}} & =e^{i m \mathbf{u} \cdot \mathbf{w} I} e^{\mathbf{w} \cdot \mathbf{P}} e^{\mathbf{u} \cdot \mathbf{k}} \\
e^{\mathbf{u} \cdot \mathbf{k}+\mathbf{w} \cdot \mathbf{P}} & =e^{\frac{i}{2} m \mathbf{u} \cdot \mathbf{w} I} e^{\mathbf{w} \cdot \mathbf{P}} e^{\mathbf{u} \cdot \mathbf{k}}
\end{aligned}
$$

Deste modo, utilizando as identidades dadas nas equações (36)-(39), obtemos

$$
\begin{aligned}
\tilde{G}^{\prime} \tilde{G}= & e^{i\left(\lambda^{\prime}+\lambda\right) I} e^{b^{\prime} H} e^{\mathbf{a}^{\prime} \cdot \mathbf{P}} e^{\mathbf{v}^{\prime} \cdot \mathbf{k}} e^{b H} e^{R^{\prime} \mathbf{a} \cdot \mathbf{P}} e^{R^{\prime} \mathbf{v} \cdot \mathbf{k}} R^{\prime} R \\
= & e^{i\left(\lambda^{\prime}+\lambda+\frac{1}{2} m \mathbf{v}^{\prime 2} b\right) I} e^{\left(b^{\prime}+b\right) H} e^{\left(\mathbf{a}^{\prime}+b \mathbf{v}^{\prime}\right) \cdot \mathbf{P}} e^{\mathbf{v}^{\prime} \cdot \mathbf{k}} \times \\
& \times e^{R^{\prime} \mathbf{a} \cdot \mathbf{P}} e^{R^{\prime} \mathbf{v} \cdot \mathbf{k}} R^{\prime} R \\
= & e^{i\left(\lambda^{\prime}+\lambda+\frac{1}{2} m \mathbf{v}^{\prime 2} b+m \mathbf{v}^{\prime} \cdot R^{\prime} \mathbf{a}\right) I} e^{\left(b^{\prime}+b\right) H} \times \\
& \times e^{\left(\mathbf{a}^{\prime}+b \mathbf{v}^{\prime}+R^{\prime} \mathbf{a}\right) \cdot \mathbf{P}} e^{\left(\mathbf{v}^{\prime}+R^{\prime} \mathbf{v}\right) \cdot \mathbf{k}} R^{\prime} R .
\end{aligned}
$$

Portanto

$$
\tilde{G}^{\prime} \tilde{G}=\left(\lambda^{\prime}+\lambda+\xi_{m}\left(G^{\prime}, G\right), G^{\prime} G\right),
$$

onde

$$
\xi_{m}\left(G^{\prime}, G\right)=m\left(\frac{1}{2} \mathbf{v}^{\prime 2} b+\mathbf{v}^{\prime} \cdot R^{\prime} \mathbf{a}\right) .
$$

Podemos verificar que a função $\xi_{m}$ obedece as equações (25) e (26), e é chamada expoente do grupo. De fato, esta função é identificada, a partir da Eq. (40), como o expoente do fator de sistema de representações unitárias projetivas. Ou seja, temos

$$
\tilde{G}\left(G^{\prime}\right) \tilde{G}(G)=w\left(G^{\prime}, G\right) \tilde{G}\left(G^{\prime} G\right),
$$

com o fator de sistema

$$
w\left(G^{\prime}, G\right)=e^{i \xi_{m}\left(G^{\prime}, G\right)} .
$$

Sintetizando, a consideração da extensão central do grupo de Galilei leva a representações unitárias irredutiveis projetivas de $\mathcal{G}$. Notemos que se $m=0, w\left(G^{\prime}, G\right)=1$, e a extensão central torna-se trivial, que é a representação fiel. Vamos então explorar a conexão 
física com o que já foi visto até aqui.

\section{EQUAÇÃO DE SCHRÖDINGER PARA PARTÍCULA LIVRE SEM SPIN}

A dedução de uma representação unitária irredutível que descreva algum sistema físico é feita identificando as equações dinâmicas invariantes pelo grupo $\tilde{G}_{\xi_{m}}$.

Tais equações podem ser dadas a partir dos invariantes da álgebra de Lie de $\tilde{G}_{\xi}$, as equações (31)-(33). Primeiro, consideramos o caso mais simples: a representação em que $H$ e $\mathbf{P}$ são escritos da forma dos operadores unitários dados pelas equações (10) e (11), os quais atuarão num espaço de Hilbert das funções escalares, $\Psi(\mathbf{x}, t)$, quadráticas integráveis dependentes de $(\mathbf{x}, t)$. Como as funções escalares são invariantes por $\tilde{G}_{\xi_{m}}$ a menos de um fator de fase $e^{i \xi_{m}\left(G^{\prime}, G\right)}$, as equações (31) - (33) nos leva a

$$
\begin{aligned}
M \Psi(\mathbf{x}, t) & =m \Psi(\mathbf{x}, t), \\
\left(H-\frac{\mathbf{P}^{2}}{2 m}\right) \Psi(\mathbf{x}, t) & =c \Psi(\mathbf{x}, t), \\
(\mathbf{J}-\mathbf{K} \times \mathbf{P})^{2} \Psi(\mathbf{x}, t) & =s^{2} \Psi(\mathbf{x}, t),
\end{aligned}
$$

onde $m, c, s^{2}$ são números que garantem a invariância destas expressões. Isto é resultado do lema de Schur, que declara que os invariantes têm valores múltiplos da identidade e rotulam uma dada representação. Uma possibilidade simples a considerar é escolher $c=s^{2}=0$ e $m \neq 0$. Desta forma, da (45) obtém-se

$$
i \frac{\partial}{\partial t} \Psi(\mathbf{x}, t)=-\frac{1}{2 m} \nabla^{2} \Psi(\mathbf{x}, t),
$$

que é justamente a equação de Schrödinger para uma partícula livre de massa $m$ e spin nulo, com $\Psi(\mathrm{x}, t)$ sendo a função de onda da partícula e $\hbar=1$. Deste modo, associamos a constante real da extensão central (30) que rotula a representação, $m$, com a massa. Podemos verificar que se $m=0$, não é possível deduzir a Eq. 47), o que nos faz afirmar que são as representações projetivas unitárias do grupo de Galilei que interessam à física não relativís- tica. O número $s^{2}$ é relacionado com o spin, e como nesta representação o spin é nulo, $s$ é zero. $\mathrm{O}$ valor $c$ é associado com com um fator de mudança no nível da energia.

É interessante notar que para nossa representação ter consistência, devemos definir o operador posição como

$$
\mathbf{Q}=\frac{\mathbf{K}}{m}-\frac{t}{m} \mathbf{P}
$$

pois a relação de $\mathbf{Q}$ com $\mathbf{P}$ gera $\left[P_{i}, Q_{j}\right]=$ $-i \delta_{i j}$, as conhecidas relações de incerteza de Heisenberg.

\section{A EQUAÇÃO DE PAULI-SCHRÖDINGER}

Nosso objetivo agora é obter uma representação unitária do grupo de Galilei estendido relacionada com uma equação para partículas não relativísticas de spin $\frac{1}{2}$, isto é, determinar uma equação análoga à de Dirac, porém no caso não relativístico.

Para tal, seguiremos a idéia que Dirac utilizou para a construção da mecânica quântica relativística. Buscamos uma equação diferencial de primeira ordem nas derivadas espaciais, $P$, e temporal, $H$. Vamos então escrever

$$
\theta \Phi=(A H+\mathbf{B} \cdot \mathbf{P}+C) \Phi=0,
$$

onde $A, \mathbf{B}$ e $C$ são objetos a serem determinados. A condição que temos é que deve existir um operador $\theta^{\prime}=\left(A^{\prime} H+\mathbf{B}^{\prime} \cdot \mathbf{P}+C^{\prime}\right)$ tal que $\theta^{\prime} \theta$ leva ao operador de Schrödinger, dado na Eq. (45), isto é

$$
\theta^{\prime} \theta=\left(H-\frac{\mathbf{P}^{2}}{2 m}\right)
$$

a menos de uma constante. Assim, os termos do produto $\theta^{\prime} \theta$ deve satisfazer:

$$
\begin{aligned}
A^{\prime} A & =0, \quad A^{\prime} B_{i}+B_{i}^{\prime} A=0, \\
A^{\prime} C+C^{\prime} A & =0, \quad B_{i}^{\prime} B_{j}+B_{j}^{\prime} B_{i}=-2 \delta_{i j},(50) \\
C^{\prime} C & =c=0, \quad C^{\prime} B_{i}+B_{i}^{\prime} C=0 .
\end{aligned}
$$


Vamos definir os operadores

$$
\begin{aligned}
& B_{4}=i\left(A+\frac{C}{2 m}\right), \quad B_{4}^{\prime}=i\left(A^{\prime}+\frac{C^{\prime}}{2 m}\right), \\
& B_{5}=A-\frac{C}{2 m}, \quad B_{5}^{\prime}=A^{\prime}-\frac{C^{\prime}}{2 m} .
\end{aligned}
$$

Logo

$$
B_{\mu}^{\prime} B_{\nu}+B_{\nu}^{\prime} B_{\mu}=-2 \delta_{\mu \nu} \quad(\mu, \nu=1, \ldots, 5) .
$$

As representações desta álgebra podem ser obtidas daquelas da álgebra de Clifford,

$$
\gamma_{\mu} \gamma_{\nu}+\gamma_{\nu} \gamma_{\mu}=2 \delta_{\mu \nu} \quad(\mu, \nu=1, \ldots, 5) .
$$

Faremos então a correspondência

$$
\begin{aligned}
& B_{\alpha}=\beta \gamma_{\alpha}, \quad B_{\alpha}^{\prime}=\gamma_{\alpha} \beta^{-1}(\alpha=1, \ldots, 4) \\
& B_{5}=-i \beta, \quad B_{5}^{\prime}=-i \beta^{-1}
\end{aligned}
$$

com $\beta$ sendo um parâmetro arbitrário. Vamos escolher, por conveniência, os resultados padrões de Dirac,

$$
\begin{aligned}
B_{i} & =\left(\begin{array}{cc}
\sigma_{i} & 0 \\
0 & \sigma_{i}
\end{array}\right) \quad(i=1,2,3), \\
B_{4} & =\left(\begin{array}{ll}
0 & 1 \\
1 & 0
\end{array}\right), B_{5}=\left(\begin{array}{cc}
0 & -i \\
i & 0
\end{array}\right),
\end{aligned}
$$

e pelas equações (51)-52 obtemos

$$
A=\left(\begin{array}{ll}
0 & 0 \\
1 & 0
\end{array}\right), C=\left(\begin{array}{cc}
0 & 2 m \\
0 & 0
\end{array}\right) \text {. }
$$

Deste modo, $\Phi=\Phi(\mathbf{x}, t)$ é um objeto a quatro componentes, que escreveremos

$$
\Phi=\left(\begin{array}{l}
\varphi \\
\chi
\end{array}\right)
$$

$\operatorname{com} \varphi$ e $\chi$ sendo funções a duas componentes. Finalmente, a Eq. (49) gera

$$
\begin{aligned}
\theta \Phi= & {\left[\left(\begin{array}{ll}
0 & 0 \\
1 & 0
\end{array}\right) H+\left(\begin{array}{cc}
\sigma \cdot \mathbf{P} & 0 \\
0 & \sigma \cdot \mathbf{P}
\end{array}\right)\right.} \\
& \left.+\left(\begin{array}{cc}
0 & 2 m \\
0 & 0
\end{array}\right)\right]\left(\begin{array}{l}
\varphi \\
\chi
\end{array}\right)=0,
\end{aligned}
$$

que pode ser reescrita como,

$$
\begin{gathered}
(\sigma \cdot \mathbf{P}) \varphi+2 m \chi=0 \\
H \varphi+(\sigma \cdot \mathbf{P}) \chi=0 .
\end{gathered}
$$

Podemos verificar, por substituição direta, que $\varphi$ e $\chi$ satisfazem a equação de Schrödinger. Portanto, a Eq. 61) descreve uma partícula não relativística com massa $m$ e spin $\frac{1}{2}$, e também é conhecida como equação de Pauli-Schrödinger. Nesta seção, introduzimos $\tilde{G}_{\xi_{m}}$ sem definir as transformações dadas nas equações (1) e (2) em um espaço vetorial métrico. Isto será considerado a seguir.

\section{COVARIÂNCIA GALILEANA}

Sabemos que o grupo de Poincaré, o grupo de simetria da física relativista, é definido como transformações isométricas lineares no espaço de Minkowski, com as transformações de Lorentz sendo vistas como rotação. A partir disto é possível dotar este espaço métrico de estrutura tensorial e desenvolver representações unitárias irredutíveis fiéis na forma manifestamente covariante.

No caso não-relativístico, não se consegue construir no espaço-tempo galileano de 4 dimensões as equações (1) e (2) como transformações lineares (do tipo rotações). Vamos então mostrar como isso pode ser implementado, o que exigirá um espaço métrico do tipo de Sitter. Dessa forma, poderemos introduzir uma formulação tensorial, e assim obter representações da álgebra de Lie de $\tilde{G}_{\xi_{m}}$ com uma abordagem explicitamente covariante. Isso abre uma gama de novas possibilidades no contexto da física da matéria condensada, através do uso dos vantajosos métodos tensoriais.

Comecemos observando que o espaço Euclidiano $\mathbb{E}$ é o espaço vetorial métrico definido no $\mathbb{R}^{3}$, tal que a distância entre dois pontos é preservada por transformações lineares. Ou seja, dado dois vetores $\mathbf{x}=\left(x^{1}, x^{2}, x^{3}\right)$ e $\mathbf{y}=\left(y^{1}, y^{2}, y^{3}\right) ; \mathbf{x}, \mathbf{y} \in \mathbb{E}$, temos

$$
r^{2}=\mathbf{x}^{2}+\mathbf{y}^{2}-2 \mathbf{x} \cdot \mathbf{y}
$$


invariante por rotações e translações. E sabemos que em sistemas físicos descritos pela física Galileana, os fenômenos obedecem às transformações em $\mathbb{E}$.

Entretanto, a transformação pura de Galilei é um tipo de translação parametrizada pelo tempo, $t$. Iremos então utilizar a distância $r$ em $\mathbb{E}$ para a introdução de um espaço métrico de dimensão maior que três, com o objetivo de inserir o parâmetro $t$ como uma coordenada, de modo que as equações (1) e (2) sejam transformações lineares nesse espaço. De fato, observamos que podemos reescrever a Eq. 62

$$
s^{2}=-\frac{1}{2} r^{2}=-t \frac{\mathbf{x}^{2}}{2 t}-t \frac{\mathbf{y}^{2}}{2 t}+\mathbf{x} \cdot \mathbf{y} .
$$

Esta expressão pode ser utilizada como o produto interno de dois vetores de um espaço penta-dimensional, denotado $\mathbb{G}$, que estabeleceremos a seguir.

Seja $\mathbb{G}$ uma variedade pseudo-Riemanniana penta-dimensional, tal que o produto interno entre dois vetores arbitrários é dado por

$$
\begin{aligned}
(x \mid y) & =\eta_{\mu \nu} x^{\mu} y^{v} \\
& =\sum_{i=1}^{3} x^{i} y^{i}-x^{4} y^{5}-x^{5} y^{4},
\end{aligned}
$$

onde $x, y \in \mathbb{G}$ (os índices latinos indicam componentes dos vetores em $\mathbb{E}$ ), e

$$
\left(\eta_{\mu \nu}\right)=\left(\begin{array}{ccccc}
1 & 0 & 0 & 0 & 0 \\
0 & 1 & 0 & 0 & 0 \\
0 & 0 & 1 & 0 & 0 \\
0 & 0 & 0 & 0 & -1 \\
0 & 0 & 0 & -1 & 0
\end{array}\right)
$$

é a métrica de $\mathbb{G}$.

A norma de um vetor em $\mathbb{G}$ é, pela Eq. (64),

$$
\|x\|=(x \mid x)=\mathbf{x}^{2}-2 x^{4} x^{5} .
$$

Assim, ocorre aqui um caso similar ao do espaço de Minkowski, pois os vetores podem ter $\|x\| \gtrless 0$ ou $\|x\|=0$. Devemos notar que se escolhemos

$$
x^{5}=\frac{\mathbf{x}^{2}}{2 t}, y^{5}=\frac{\mathbf{y}^{2}}{2 t} \text { e } x^{4}=y^{4}=t,
$$

então $s^{2}$ na Eq. (63) é um caso particular do produto interno em $\mathbb{G}$; além disso, observamos que a norma de $x$, na Eq. (66), é nula. O ajuste de unidades físicas é feito tomando $x^{4}=y^{4}=$ $v t, \operatorname{com} v=1 \mathrm{~m} / \mathrm{s}$. Deste modo, vetores em $\mathbb{G}$ associados com a invariânia Galileana têm norma nula.

Podemos expressar esta correspondência através de uma imersão do espaço Euclidiano em $\mathbb{G}$, onde todo vetor $\mathbf{A} \in \mathbb{E}$ é imerso nesse espaço por

$$
\Im: \mathbf{A} \longmapsto A=\left(\mathbf{A}, A_{4}, \frac{\mathbf{A}^{2}}{2 A_{4}}\right) ; A \in \mathbb{G} .
$$

A estrutura tensorial deste espaço métrico tem implementação imediata, tomando $\left\{e_{\mu}\right\}(\mu=1, \ldots, 5)$ como a base do espaço tangente de $\mathbb{G}$ e $\left\{e^{\mu}\right\}$ a base do espaço cotangente, $T_{p}^{*} \mathbb{G}$. Considerando um vetor arbitrário $X$ em $\mathbb{G}$, e escrevendo-o em componentes da base $\left\{e_{\mu}\right\}$ de $T_{p}^{*} \mathbb{G}$, implica em $X=X^{\mu} e_{\mu}$. Utilizando a métrica, relacionamos as componente contravariante e covariantes (ou seja, definimos o levantamento e abaixamento dos índices) de modo usual, $\left(\eta^{\mu \nu}\right)^{-1}=\eta_{\mu \nu}$, então $X^{\mu}=\eta^{\mu \nu} X_{\nu}$, que resulta em

$$
\begin{aligned}
& X^{i}=X_{i}, \text { se } i \leq 3 ; \\
& X^{4}=-X_{5}, \\
& X^{5}=-X_{4} .
\end{aligned}
$$

De modo similar temos $X_{\nu}=\eta_{\mu \nu} X^{\mu}$.

Em $\mathbb{G}$ um tensor do tipo $J_{r, p}^{q}(\mathbb{G})$ é definido pelo mapeamento 81 .

$$
\begin{aligned}
& T: T_{p}^{*} \mathbb{G}^{(1)} \otimes \ldots \otimes T_{p}^{*} \mathbb{G}^{(q)} \otimes T_{p} \mathbb{G}^{(1)} \otimes \ldots \\
& \otimes T_{p} \mathbb{G}^{(r)} \longmapsto \mathbb{R},
\end{aligned}
$$

tal que $T$ é escrito como

$$
T=T_{\nu_{1} \ldots \nu_{r}}^{\mu_{1} \ldots \mu_{q}} e_{\mu_{1}} \otimes \ldots \otimes e_{\mu_{q}} \otimes e^{\nu_{1}} \otimes \ldots \otimes e^{\nu_{r}},
$$

com as componentes dadas por

$$
T^{\mu_{1} \ldots \mu_{q}} \nu_{\nu_{1} \ldots \nu_{r}}=T\left(e^{\mu_{1}}, \ldots, e^{\mu_{q}}, e_{\nu_{1}}, \ldots, e_{\nu_{r}}\right) .
$$

É interessante agora analisar as transformações isométricas em $\mathbb{G}$. Considere um 
conjunto de transformações lineares do tipo

$$
\overline{\mathbf{x}}^{\mu}=G^{\mu} x^{\nu}+a^{\mu},
$$

com $|G|=1$. Vamos determinar os geradores dessas transformações, utilizando transformações infinitesimais dadas por

$$
G_{\nu}^{\mu}=\delta_{\nu}^{\mu}+\epsilon_{\nu}^{\mu} .
$$

Utilizando a representação unitária sobre o espaço das funções de um ponto em $\mathbb{G}$, temos

$$
\begin{aligned}
M_{\mu \nu} & :=-i\left(x_{\mu} \partial_{\nu}-x_{\nu} \partial_{\mu}\right), \\
P_{\mu} & :=-i \partial_{\mu}
\end{aligned}
$$

onde $M_{\mu \nu}$ 's são os geradores das transformações homogêneas e os $P_{\mu}$ 's das nãohomogêneas.

Esses geradores satisfazem a seguinte álgebra de Lie

$$
\begin{aligned}
{\left[M_{\mu \nu}, M_{\rho \sigma}\right]=} & -i\left(\eta_{\nu \rho} M_{\mu \sigma}-\eta_{\mu \rho} M_{\nu \sigma}\right. \\
& \left.+\eta_{\mu \sigma} M_{\nu \rho}-\eta_{\nu \sigma} M_{\mu \rho}\right), \\
{\left[P_{\mu}, M_{\rho \sigma}\right]=} & -i\left(\eta_{\mu \rho} P_{\sigma}-\eta_{\mu \sigma} P_{\rho}\right) \\
{\left[P_{\mu}, P_{\nu}\right]=} & 0 .
\end{aligned}
$$

As equações (78)- (80) formam uma álgebra de Lie $\mathfrak{g}$, e que tem forma similar à álgebra do grupo de Poincaré.

Como um exemplo preliminar, vamos analisar um caso particular das transformações (74) em $\mathbb{G}$. Considere a transformação

$$
\begin{aligned}
& \bar{x}^{i}=R_{j}^{i} x^{j}+v^{i} x^{4}+a^{i}, \\
& \bar{x}^{4}=x^{4}+a^{4}, \\
& \bar{x}^{5}=x^{5}+v_{i}\left(R_{j}^{i} x^{j}\right)+\frac{1}{2} \mathbf{v}^{2} x^{4} .
\end{aligned}
$$

$\mathrm{Na}$ representação matricial, as transformações homogêneas são escritas por

$$
\left(G^{\mu}{ }_{\nu}\right)=\left(\begin{array}{ccccc}
R_{1}^{1} & R_{2}^{1} & R_{3}^{1} & v^{1} & 0 \\
R_{1}^{2} & R_{2}^{2} & R_{3}^{2} & v^{2} & 0 \\
R_{1}^{3} & R_{2}^{3} & R_{3}^{3} & v^{3} & 0 \\
0 & 0 & 0 & 1 & 0 \\
v_{i} R_{1}^{i} & v_{i} R_{2}^{i} & v_{i} R_{3}^{i} & \frac{1}{2} \mathbf{v}^{2} & 1
\end{array}\right) .
$$

Seguindo a mesma idéia anterior, analisando transformações infinitesimais para as equações
(81)- 83) como aquelas descritas na Eq. 757, podemos reescrever os geradores dados na Eq. (76) como

$$
\begin{aligned}
J_{i} & =\frac{1}{2} \varepsilon_{i j k} M_{j k}, \\
K_{i} & =M_{5 i} \\
C_{i} & =M_{4 i}, \\
D & =M_{54} .
\end{aligned}
$$

As relações (78)-(80), considerando apenas aquelas não-nulas, são reescritas como

$$
\begin{array}{rlrl}
{\left[J_{i}, J_{j}\right]} & =i \varepsilon_{i j k} J_{k}, & & {\left[J_{i}, K_{j}\right]=i \varepsilon_{i j k} K_{k},} \\
{\left[J_{i}, C_{j}\right]=i \varepsilon_{i j k} C_{k},} & & {\left[K_{i}, C_{j}\right]=i \delta_{i j} D} \\
{\left[D, K_{i}\right]} & =i K_{i}, & & +i \varepsilon_{i j k} J_{k}, \\
{\left[P_{4}, D\right]} & =i P_{4}, & & {\left[C_{i}, D\right]=i C_{i},} \\
{\left[P_{i}, K_{j}\right]=i \delta_{i j} P_{5},} & & {\left[J_{i}, P_{j}\right]=i \varepsilon_{i j k} P_{k},} \\
{\left[P_{4}, K_{i}\right]=i P_{i},} & & {\left[P_{i}, C_{j}\right]=i \delta_{i j} P_{4},} \\
{\left[D, P_{5}\right]} & =i P_{5}, & & {\left[P_{5}, C_{i}\right]=i P_{i} .}
\end{array}
$$

Estas relações formam uma álgebra que tem como subálgebra a álgebra de Lie do grupo de Galilei, considerando $J_{i}$ os geradores das rotações, $K_{i}$ e $C_{i}$ das transformações puras, $P_{\mu}$ das translações espaciais e temporais, e $D$ do tipo dilatação temporal. E de fato, podemos observar que as equações (81) e (82) são justamente as transformações de Galilei dadas pelas equações (1) e (2), com $x^{4}=t$. A Eq. (83) é uma condição de compatibilidade que representa a imersão $\Im$, pois por coerência a norma deste tipo de vetor em $\mathbb{G}$ tem de ser nula. A comutação entre $K_{i}$ e $P_{j}$ é naturalmente diferente de zero neste contexto, com $P_{5}$ sendo relacionado à massa. Neste contexto, o estudo das representações unitárias irredutíveis fiéis do grupo de Galilei $\mathbb{G}$ é que tem interesse para a física Galileana, e por construção fica semelhante ao caso do grupo de Poincaré para a física relativista. Observe que as transformações dadas pelas equações (81)83 deixam a métrica (ou o produto escalar) invariantes.

\section{- Significado físico das 5 dimensões}

Um vetor de interesse em $\mathbb{G}$ é o 5 momentum, $p^{\mu}=(\mathbf{p}, m, E)$, sendo que $E=$ 
$\mathbf{p}^{2} / 2 m$, o que leva $p^{\mu}$ a estar de acordo com a imersão $\Im$, e pelas equações (81)-(83) se transforma como $\bar{p}^{\mu}=G_{\nu}^{\mu} p^{\mu}$, ou seja,

$$
\begin{aligned}
& \bar{p}^{i}=R_{j}^{i} p^{j}+v^{i} p^{4}, \\
& \bar{p}^{4}=p^{4}, \\
& \bar{p}^{5}=v_{i}\left(R_{j}^{i} p^{j}\right)+\frac{1}{2} \mathbf{v}^{2} p^{4}+p^{5},
\end{aligned}
$$

considerando apenas as transformações homogêneas. Escrevendo $p^{\mu}$ infinitesimalmente

$$
d p^{2}=(d \mathbf{p})^{2}-2 d p^{4} d p^{5}=d k^{2} .
$$

Assim as 5 componentes de $p$ descrevem: as 3 componentes do momentos lineares $(d \mathbf{p})$, a massa $\left(d p^{4}\right)$ e a energia $\left(d p^{5}\right)$ (a menos de um fator de velocidade fixando as unidades). As coordenadas canonicamente conjugadas são dadas pelo 5 -vetor $x$, de tal modo que

$$
d x^{2}=(d \mathbf{x})^{2}-2 d x^{4} d x^{5}=d s^{2} .
$$

As 5 componentes descrevem: 3 coordinadas em $\mathbb{E}(d \mathbf{x})$; uma coordenada descreve o tempo, desde que $d x^{4}=d t$, e uma coordenada descreve projeção da velocidade, desde que

$$
d x^{5}=\frac{(d \mathbf{x})^{2}}{2 d t}=\frac{1}{2} d \mathbf{x} \cdot \mathbf{v}
$$

(a menos de um fator de velocidade fixando as unidades).

\section{- Transformações de operadores e in- variantes}

Nesta abordagem, os operadores diferenciais $\partial_{\mu} \equiv \frac{\partial}{\partial x^{\mu}}$ também se transformam como vetores, isto é,

$$
\bar{\partial}_{\mu}=G_{\mu}{ }^{\nu} \partial_{\nu}
$$

$\operatorname{com} G_{\mu}{ }^{\nu}=\eta_{\mu \rho} G_{\sigma}^{\rho} \eta^{v \sigma}$, dado por

$$
\left(G_{\mu}{ }^{\nu}\right)=\left(\begin{array}{ccccc}
R_{1}^{1} & R_{2}^{1} & R_{3}^{1} & 0 & -v^{1} \\
R_{1}^{2} & R_{2}^{2} & R_{3}^{2} & 0 & -v^{2} \\
R_{1}^{3} & R_{2}^{3} & R_{3}^{3} & 0 & -v^{3} \\
-v_{i} R_{1}^{i} & -v_{i} R_{2}^{i} & -v_{i} R_{3}^{i} & 1 & \frac{1}{2} \mathbf{v}^{2} \\
0 & 0 & 0 & 0 & 1
\end{array}\right) .
$$

Observamos das equações (92) e (93) que $\bar{\partial}_{\mu} \bar{\partial}^{\mu}=\partial_{\mu} \partial^{\mu}$ e $\bar{\partial}_{5}=\partial_{5}$, e fazendo a correspondência com os geradores $P_{\mu}$ 's, logo

$$
\begin{aligned}
& I_{1}=P_{\mu} P^{\mu}, \\
& I_{2}=P_{5},
\end{aligned}
$$

são os invariantes de Casimir da álgebra de Lie relacionada ao grupo de Galilei. Cabe ressaltar que existe um terceiro invariante, obtido via vetor de Pauli-Lubanski em $\mathbb{G}$. Este invariante carrega a informação do spin.

\section{REPRESENTAÇÕES EM $\mathbb{G}$}

Seguindo a idéia da seção anterior, partindo dos invariantes (94) e (95), podemos deduzir representações unitárias irredutíveis que descrevem partículas quânticas livres.

\section{- Equação de Schrödinger: repre- sentação escalar}

Considerando o caso mais simples, temos $I_{1}$ e $I_{2}$ sobre o espaço das funções escalares $\Phi(x)$ dependentes de $x \in \mathbb{G}$. Sob as transformações (81)-83), obtemos $\bar{\Phi}(\bar{x})=\Phi(x)$. Assim, $I_{1} \mathrm{e}$ $I_{2}$ atuando nestas funções tem que dar como resultado uma quantidade igualmente invariante. Com a correspondência $P_{\mu}=-i \partial_{\mu}$, podemos escrever as expressões

$$
\begin{aligned}
-\partial_{\mu} \partial^{\mu} \Phi(x) & =k^{2} \Phi(x), \\
\partial_{5} \Phi(x) & =-i m \Phi(x),
\end{aligned}
$$

onde $k$ é uma constante positiva e $m$ outra constante que pode ser associada à massa. Considerando $k=0$, obtemos

$$
\left(-\nabla^{2}+2 \partial_{4} \partial_{5}\right) \Psi=0 .
$$

Porém, a Eq. 97) nos diz que $\Phi(x)$ é da forma

$$
\Phi(x)=e^{-i m x^{5}} \Psi\left(\mathbf{x}, x^{4}\right) .
$$

Substituindo este resultado na Eq. (98), obtemos

$$
i \partial_{4} \Psi\left(\mathbf{x}, x^{4}\right)=-\frac{1}{2 m} \nabla^{2} \Psi\left(\mathbf{x}, x^{4}\right),
$$


que é igual a Eq. (47) com $\partial_{4}=\partial_{t}$. Assim temos a equação de Schrödinger, onde $\Psi\left(\mathbf{x}, x^{4}\right)$ descreve a função de onda de uma partícula livre.

\section{- Equação de Pauli-Schrödinger: re- presentação spinorial}

Vamos tratar agora da construção de uma equação de onda para as partículas com spin $\frac{1}{2}$. Para isso podemos utilizar o prodecimento de Dirac, mas agora de modo covariante, e escrever uma equação de primeira ordem nas derivadas do tipo

$$
\left(i \gamma^{\mu} \partial_{\mu}-k\right) \Phi=0
$$

onde $\gamma^{\mu}$ são entes a serem determinados e $\Phi$ é a função de onda da partícula; esta equação deve ser tal que multiplicada por $i \gamma^{\mu} \partial_{\mu}$ gere a Eq. (100). Logo, multiplicando a Eq. 101 por $\left(i \gamma^{\mu} \partial_{\mu}+k\right)$, obtemos

$$
\left(-\gamma^{\nu} \gamma^{\mu} \partial_{\nu} \partial_{\mu}-i k \gamma^{\nu} \partial_{\nu}\right) \Phi=0 .
$$

Para que esta expressão leve à equação de Schrödinger, os entes $\gamma^{\mu}$ devem obedecer a álgebra de Clifford, ou seja,

$$
\gamma^{\nu} \gamma^{\mu}=\frac{1}{2}\left(\gamma^{\nu} \gamma^{\mu}+\gamma^{\mu} \gamma^{\nu}\right)=\frac{1}{2}\left\{\gamma^{\nu}, \gamma^{\mu}\right\}=\eta^{\nu \mu}
$$

E com a Eq. 101, a Eq. (102) torna-se

$$
\left(-\eta^{\nu \mu} \partial_{\nu} \partial_{\mu}-k^{2}\right) \Phi=0,
$$

ou seja a Eq. (96). Deste modo, utilizaremos, por conveniência uma representação na qual os entes $\gamma^{\mu}$ são dados por

$$
\begin{aligned}
\gamma^{i} & =\left(\begin{array}{cc}
\sigma_{i} & 0 \\
0 & -\sigma_{i}
\end{array}\right), \gamma^{4}=\left(\begin{array}{cc}
0 & 0 \\
-\sqrt{2} & 0
\end{array}\right), \\
\gamma^{5} & =\left(\begin{array}{cc}
0 & \sqrt{2} \\
0 & 0
\end{array}\right),
\end{aligned}
$$

onde $\sigma_{i}$ são as matrizes de Pauli e $\sqrt{2}$ é a matriz identidade $2 \times 2$ multiplicada por $\sqrt{2}$. O objeto $\Phi$ terá quatro componentes, da mesma forma que na Eq. 60) (mas com $\varphi$ e $\chi$ dependentes de $\left.x^{\mu} ; \mu=1, \ldots, 5\right)$. Portanto, na representação em que $k=0$, a Eq.(101) se reduz a

$$
\begin{gathered}
\sigma \cdot \nabla \varphi+\sqrt{2} \partial_{5} \chi=0, \\
-\sqrt{2} \partial_{4} \varphi-\sigma \cdot \nabla \chi=0
\end{gathered}
$$

e é assim, como a Eq.61), uma equação de onda não relativística que descreve partículas com spin $\frac{1}{2}$ e massa $m$.

A consequência dessa formulação covariante é que todo aparato teórico desenvolvido no caso relativístico pode ser explorado no caso não-relativístico. Por exemplo, a densidade de Lagrangiana para a equação de Pauli-Schrödinger covariante é dada por

$$
L=\bar{\Phi}\left(i \gamma^{\mu} \partial_{\mu}-k\right) \Phi,
$$

similar ao caso relativístico. Podemos então introduzir, conduzidos pela covariância, termos de interação não lineares, explorar as transformações de calibre abelianos ou nãoabelianos, etc. São esses aspectos que têm sido desenvolvidos na literatura, e aberto novas perspectivas para a física da matéria condensada, de um ponto de vista teórico [56, 57].

\section{CONCLUSÕES E OBSERVAÇÕES FINAIS}

Neste trabalho apresentamos uma revisão breve sobre o grupo de Galilei e desenvolvemos sua estrutura tensorial subjacente. Assim construimos um espaço-tempo $\mathbb{G}$ onde as transformações de Galilei são obtidas por transformações unitárias. Por uma transformação de coordenadas, $x^{4} \rightarrow\left(x^{4}-x^{5}\right) / \sqrt{2}$ e $x^{5} \rightarrow$ $\left(x^{4}+x^{5}\right) / \sqrt{2}$, com $x^{i}$ mantidos invariantes, temos que

$$
x^{2}=\mathbf{x}^{2}-2 x^{4} x^{5} \rightarrow \mathbf{x}^{2}-\left(x^{4}\right)^{2}+\left(x^{5}\right)^{2} .
$$

Ou seja, a métrica fica diagonal, e passa ser escrita como

$$
\left(\eta_{\mu \nu}\right)=\left(\begin{array}{ccccc}
1 & 0 & 0 & 0 & 0 \\
0 & 1 & 0 & 0 & 0 \\
0 & 0 & 1 & 0 & 0 \\
0 & 0 & 0 & -1 & 0 \\
0 & 0 & 0 & 0 & 1
\end{array}\right) .
$$


Isto significa que $\mathbb{G}$ é o espaço de de Sitter em $(4+1)$ dimensões. A física Galileana é obtida quando fazemos a imersão no cone de luz desse espaço. Observe que no caso da física relativística, para se obter resultados físicos, devemos fazer uma imersão para dentro do cone de luz no espaço de Minkowski em $(3+1)$ dimensões.

Como a física não relativística é obtida no cone-de-luz, o grupo mais geral que deixa as equações de movimento invariante é o grupo conforme, definido em $\mathbb{G}$. Este fato tem sido explorado recentemente para o desenvolvimento de teorias não relativística duais do tipo AdS/CFT, para tratar com sistemas de spins $1 / 2$ [56 58].

\section{Agradecimentos}

AES agradece ao IF-UFBA pela hospitalidade, onde parte significativa deste trabalho foi desenvolvido. Este trabalho recebeu apoio financeiro parcial do CNPq.
[1] H. Weyl, Symmetry. New Jersey: Princeton University Press (1980).

[2] R. Bourgue, J-P Azra, (Eds.), Oeuvres $d$ 'Evariste Galois. Paris: Gauthier-Villars (1962).

[3] S. Lie. Math. Annalen 5, 145 (1872).

[4] L. Dresner, Applications of Lie's Theory of Ordinary and Partial Differential Equations. Bristol: IoP (1999).

[5] P.J. Olver, Applications of Lie Groups to Differential Equations. New York: SpringerVerlag (1993).

[6] A. Fazzio, K. Watari, Introdução à Teoria dos Grupos. Santa Maria: EdUFSM (1997).

[7] J.D.M. Vianna, Teoria de Grupos Aplicados a Física. Notas de Curso. Brasília: IF-UnB (1983).

[8] R. Gilmore, Lie Groups, Lie Algebras and Some of their Applications. New York: J. Wiley (1975) (Reedited by Dover, 2005).

[9] W.I. Fushchich, A. G. Nikitin, Symmetries of Equations of Quantum Mechanics. New York: Allerton Press (1994).

[10] E.P. Wigner. Ann. Math. 40, 149 (1939).

[11] A. Aurilia, H. Umezawa. Phys. Rev. 182, 1682 (1969).

[12] S.S. Schweber, An Introduction to Relativistic Quantum Field Theory. London: Harper-Row (1961).

[13] I.M. Gel'Fand, R.A. Minlos, Z.Ya. Shapiro, Representations of the Rotation and Lorentz Groups. New York: Pergamon Press (1963).

[14] C.N. Yang, R.L. Mills. Phys. Rev. 96, 191 (1954).

[15] M. Hamermesh, Group Theory and its Application to Physical Problems. New York: Dover (1989).

[16] A.E. Santana. Rev. Bras. Ens. Fis. 19, 113
(1997)

[17] E. Inönü, E.P. Wigner. N. Cimento 9, (1952) 705. (Uma apresentação interessante sobre a importância pedagógica e epistemológica da teoria de grupo em mecânica quântica nãorelativista pode ser encontrado em: J.M. LévyLeblond. N. Cimento 4, 99 (1974).)

[18] V. Bargmann. Ann. Math. 59, 1 (1954).

[19] Os resultados sobre as representações projetivas para o grupo de Galilei apareceram primeiro numa comunicação por Bargmann, não publicada e citada no artigo de Inönü e Wigner (Ref.6).

[20] M. Hamermesh. Ann. Phys. (N.Y.) 17, 518 (1960).

[21] J.M. Levy-Leblond. J. Math. Phys. 4, 776 (1963).

[22] A. Loinger. Ann. Phys. (N.Y.) 20, 132 (1962).

[23] J.M. Levy-Leblond. Comm. Math. Phys. 4, 157 (1967).

[24] J.M. Levy-Leblond. Comm. Math. Phys. 6, 286 (1967).

[25] H. Bacry, J.M. Levy-Leblond. J. Math. Phys. 9, 1605 (1968).

[26] J. Voisin. J. Math. Phys. 8, 611 (1967).

[27] R. Pauri. J. Math. Phys. 9, 1146 (1968).

[28] C.R. Hagen, W.J. Hurley. Phys. Rev. Lett. 24, 1381 (1970).

[29] C.R. Hagen. Phys. Rev. D 5, 377 (1972).

[30] P.R. Roman, J.J. Aghassi, R.M. Santilli, P.L. Huddeleston. N. Cimento 12A, 185 (1972).

[31] E.C.G. Sudarshan, N. Mukunda, Classical Dynamics: A Modern Perspective. New York: John Wiley (1974).

[32] L. Gagnon. Can. J. Phys. 67, 1 (1989).

[33] L. Gagnon, P. Winternitz., J. Phys. A: Math. Gen. 21, 1493 (1988). L. Gagnon, P. Winternitz. J. Phys. A: Math. Gen. 22, 469 (1989). 
L. Gagnon, P. Winternitz. J. Phys. A: Math. Gen. 22, 499 (1989).

[34] H. Umezawa, Advanced Field Theory: Micro, Macro and Thermal Physics. New York: AIP (1993).

[35] A.E. Santana, A. Matos Neto, J.D.M. Vianna. Hadronic J. 17, 539 (1994).

[36] A.E. Santana, F.C. Khanna. Phys. Lett. A203, 68 (1995).

[37] A.E. Santana, F.C. Khanna, Lie Groups and Thermofield Dynamics. In: Proceedings of the Dalian-THERMO95. Y.X. Gui, F.C. Khanna, Z.B. Su (Eds.). Singapore: World Scientific (1996).

[38] A.E. Santana, F.C. Khanna, H. Chu, Y.C. Chang. Ann. Phys. (N.Y) 249, 481 (1996).

[39] A. Matos Neto, A.E. Santana, J.D.M. Vianna, F.C. Khanna. Physica A 280, 405 (2000).

[40] F.C. Khanna, A.P.C. Malbouisson, J.M.C. Malbouisson, A.E. Santana, Thermal Quantum Field Theory: Algebraic Aspects and Applications. Singapore: World Scientific (2009).

[41] L.D. Landau. J. Phys. (Moscou) 5, 71 (1941).

[42] Y. Takahashi. Fortschr. Phys. 36, 63 (1988).

[43] Y. Takahashi. Fortschr. Phys. 36, 83 (1988).

[44] M. Omote, et al.. Fortschr. Phys. 37, 933 (1989).

[45] A.E. Santana, F.C. Khanna, Y. Takahashi. Prog. Theor. Phys. 99, 327 (1998).

[46] M. de Montigny, F.C. Khanna, A.E. Santana, E.S. Santos, J.D.M. Vianna. Ann. Phys. (N.Y.) 277, 144 (1999).

[47] M. de Montigny, F.C. Khanna, A.E. Santana, E.S. Santos, J.D.M. Vianna. J. Phys. A: Math. Gen. 33, (31) L273 (2000).

[48] L.M. Abreu, A.E. Santana, A. Ribeiro Filho. Ann. Phys. (N.Y.) 297, 396 (2002).

[49] B. Carter, N. Chamel. Int. J. Mod. Phys. D 13, 291 (2004). [ArXiv: astro-ph/0305186].

[50] B. Carter, N. Chamel. Int. J. Mod. Phys. D 14, 717 (2005). [ArXiv: astro-ph/0312414].

[51] B. Carter, N. Chamel. Int. J. Mod. Phys. D 14, 749 (2005). [ArXiv: astro-ph/0410660].

[52] H.P. Kunzle, C. Duval. Class. Quant. Grav. 3, 957 (1986).

[53] H.P. Kunzle. Gen. Rel. Grav. 7, 445 (1976).

[54] S.C. Ulhoa, F.C. Khanna, A.E. Santana, Galilean Covariance and the Gravitational Field. Int. J. Mod. Phys. A 24, 5287 (2009). [ArXiv:gr-qc 0902.2023].

[55] M. Kobayashi, M. de Montigny, F.C. Khanna. Phys. Lett. A 372, 3541 (2008).

[56] D.T. Son. Phys. Rev. D 78, 046003 (2008).

[57] K. Balasubramanian, J. McGreevy. Phys. Rev. Lett. 101, 061601 (2008).
[58] D. Yamada, Thermodynamics of Black Holes in Schrödinger Space. [ArXiv:hep-th/0809.4928 (2008)].

[59] K.M. O 'Hara et al.. Science 298, 2179 (2002).

[60] T. Bourdel et al.. Phys. Rev. Lett. 93, 050401 (2004).

[61] G. Galilei, Dialogue Concerning the Two Chief World Systems. 2nd Ed. Translated by Stillman Drake. Berkeley: Univ. of California Press (1967). G. Galilei, Dialogo Di Galileo Galilei Linceo Doue ne I congreffi di Quattro giornate fi difcorre fopra I due Massimi Sistemi Del Mondo Tolemaico, e Copernicano. In: Fiorenza, Per Gio: Batifta Landini MDCXXXII (1632).

[62] G. Galilei, Dialogue Concerning the Two New Sciences. Translated by Henry Crew and Alfonso di Salvio. New York: Dover (1954).

[63] G. Galilei, A Mensagem das Estrelas. Rio de Janeiro: Museu de Astronomia e Ciências Afins (1987).

[64] G. Galilei, Cartas. Discussões. Diálogos. Lisboa: Delfos (1970).

[65] D. Shapere, Galileo - A Philosophical Study. Chicago: The University of Chicago Press (1974).

[66] D. Sobel, A Filha de Galileu - Um Relato Biográfico de Ciência, Fé e Amor. Tradução por Eduardo Brandão. São Paulo: Companhia das Letras (2000).

[67] No seu livro Galileo's daughter, a sua autora Dara Sobel transcreve no capítulo XIV a citação de Salviati mencionada na introdução deste trabalho, baseada na mesma edição inglesa da mencionada obra de Galilei. Este trecho foi traduzido para a edição brasileira [66]: "Tranque-se com um amigo na cabine principal sob o convés de um grande navio $e$ leve consigo algumas moscas, borboletas e outros pequenos animais voadores. Leve também um grande vaso d'água com um peixe dentro; pendure uma garrafa que se esvazie gota a gota dentro de uma larga vasilha debaixo dela. Com o navio parado, observe cuidadosamente como os pequenos animais voam com velocidade idêntica para todos os lados da cabine. $O$ peixe nada indiferentemente em todas as direções; as gotas caem dentro da vasilha embaixo; e, ao jogar alguma coisa para seu amigo, você não precisa jogá-la com mais força numa direção do que na outra, sendo iguais as distâncias. Depois de observar todas essas coisas cuidadosamente (embora não haja dúvida de que, quando o navio está parado, tudo acontece desse modo), faça 
o navio mover-se na velocidade que você desejar, contanto que o movimento seja uniforme, sem nenhum tipo de variação. Você não vai descobrir a mais ínfima mudança em todos os efeitos citados, nem poderá dizer, baseado em qualquer deles, se o navio estava se movendo ou parado".

[68] P.N. Mariconda (Org.), O Pensamento Vivo de Galileu. São Paulo: Martin Claret (1987).

[69] J.M. Knudsen, P.G. Hjorth, Elements of Newtonian Mechanics. Berlin: Springer Verlag (1996).

[70] Alguns autores têm enfatizado o trecho do livro citado na Introdução como a gênese da relatividade galileana. Claret 68] faz uma tradução livre do mesmo. J.M. Knudsen e P.G. Hjorth 69, pp. 90-91, na sua discussão sobre "The Galilei Transformation" transcreve o mesmo trecho do diálogo entre Salviati e Sagredo na seção 4.2 - "Galileo Speaks ..."

[71] Galileu Galilei nasceu em Pisa em 15/02/1564 e faleceu em Arcetri em 08/01/1642. Era filho de Vicêncio Galilei e Júlia di Cosimo degli Ammanati. Infelizmente, em vista de dificuldades financeiras da família, Galileu não conseguiu completar um curso de estudos humanísticos. Ao mesmo tempo que estudava o latim e o grego trabalhava como aprendiz de tecelão. O domínio da língua latina proporcionou-lhe o acesso à leitura de filósofos e cientistas e também escrever as suas mais importantes obras, além de possibilitar correspondência com os eruditos da Europa. Foi iniciado ao estudo da Lógica pelo Padre Valombrosano, entretanto, por interesse paterno ele deveria seguir Medicina. Matriculouse então na Universidade de Pisa onde estudou Filosofia e Matemática, disciplinas necessárias à citada carreira (àquela época). Era um insatisfeito com os seus mestres por intensificarem as doutrinas de Aristóteles, Platão e outros filósofos antigos. Aos 18 anos estudou o isocronismo do pêndulo físico, observando as oscilações de uma lâmpada suspensa no teto da catedral de Pisa. Neste mesmo período solicitou ao padre Ostilio Ricci di Fermo, amigo de sua família, ensinamentos sobre a Geometria de Euclides. Mesmo desistindo da Medicina e não mais recebendo as lições euclidianas, ele prosseguiu com tais estudos e com aqueles de Arquimedes. Decidiuse pela Matemática e graças à ajuda do seu amigo Marquês Guidobaldo del Monte, que o recomendou, obteve a cátedra de Matemática em Pisa, aos 25 anos, por um período de três anos. Além das dificuldades financeiras, o trabalho de professor não era bem remunerado, ele ainda não gozava da simpatia de seus velhos colegas professores. Foi na Universidade de Pisa, em 1591, que ele demonstrou, pela primeira vez, perante alunos e mestres, que todos os corpos, pesados ou leves, caem com igual velocidade, demonstrando publicamente, segundo a lenda, na torre inclinada de Pisa. Graças à ajuda de seu amigo del Monte e do nobre florentino Filipe Salviati (homenageado com o Salviati nos Diálogos ...) ele em 26 de setembro de 1592, foi nomeado professor de Matemática da Universidade de Pádua, com um contrato por seis anos. Ressalte-se que um outro seu amigo Gianfrancesco Sagredo, de Veneza, que tornou-se outro seu personagem, no célebre livro, também o ajudou na obtenção daquele posto universitário.

[72] Nicolau Copérnico (1473 - 1543), astrônomo e cônego polaco, introduziu na sua obra seminal "De Revolutionibus Orbium Coelestium" (Das Revoluções dos Orbes Celestes), publicado no ano de seu perecimento. Com o seu heliocentrismo, segundo Bassalo 73, ele retomou a idéia de um universo heliocêntrico defendido, anteriormente, por Aristarco de Samos (320 250), Oresme (1325 - 1382) e Nicolau de Cusa (1401 - 1464). De acordo com esse modelo a Terra é imóvel e gira em torno do Sol.

[73] J.M.F. Bassalo, Crônicas da Física - Tomo 1. Belém: GEU-UFPA (1987).

[74] Cláudio Ptolomeu (85-165) introduziu o modelo de sistema planetário que leva o seu nome. De acordo com o mesmo, a Terra permanece imóvel e era o centro do sistema de planetas em que vivemos.

[75] De acordo com Nascimento 76] a presença de "linceu" no título dos "Diálogos ..." é para indicar que o autor era membro da Academia dos Linceus, fundada pelo príncipe Frederico Cesi, em 1603. Na segunda jornada do Diálogos, centraliza-se em neutralizar os argumentos tradicionais contra a rotação diurna da Terra. Para conseguir isto, Galilei introduz o princípio da relatividade do movimento, bem como, o de conservação (inércia). Estes, na realidade, subvertem as próprias bases da Física aristotélica e constituem o único meio de descartar os argumentos contra a rotação, que são irrefutáveis dentro do quadro aristotélico da Física ...".

[76] C.A.R. Nascimento, Para Ler Galileu Galilei - Diálogo Sobre os Dois Máximos Sistemas do Mundo. São Paulo: Nova Stella-EDUC (1990). 
[77] Na relatividade galileana clássica, a lei de composição de velocidades explicita que "a velocidade de um corpo, em relação a um outro em repouso, é igual à velocidade que ele apresenta em relação a um outro corpo que se desloca com velocidade constante em relação ao corpo em repouso, acrescida desta última velocidade". Foi a partir desta lei e da lei da inércia, incluídas em 1632 por Galilei no seu Diálogos ..., que Isaac Newton (1642-1727) elaborou o conceito do que seria consagrado como um referencial inercial.

[78] M. Gleiser, A Dança do Universo - dos Mitos de Criação ao Big-Bang. São Paulo: Companhia das Letras (1997).

[79] O filósofo italiano Giordano Bruno (15481600) propôs a realização de experiências que poderiam ser efetuadas a bordo de um navio em movimento uniforme. Ele com isso conseguiu responder às críticas dos aristotélicos que não aceitavam o movimento da Terra.
Bruno é também reconhecido como um dos precursores do princípio da independência dos movimentos, ou princípio da relatividade clássica, ou galileana do movimento, ou princípio da relatividade clássica de Giordano Bruno-Galileu-Newton 73 .

[80] Bassalo 73 cita Nicole d'Oresme (1325-1382) que foi diretor do Colégio de Navarra da Universidade de Paris e realizou inúmeros trabalhos importantes, sendo inclusive um dos iniciadores da Geometria Analítica desenvolvida por René Descartes e publicada em 1637. Ele demonstrou a regra mertoniana "num movimento uniformemente acelerado (diforme) a distância percorrida aumenta com o quadrado do tempo". Apesar disso, ele não conseguiu enunciar a lei da queda livre dos corpos, o que seria feito por Galilei, em 1638.

[81] M. Nakahara, Geometry, Topology and Physics. Bristol: IoP (1990). 